\title{
On the consistency of the current conventional EOP series and the celestial and terrestrial reference frames
}

\author{
Santiago Belda ${ }^{1,2,3}$ - Robert Heinkelmann ${ }^{3}$ - José M. Ferrándiz ${ }^{2}$. Tobias Nilsson ${ }^{3}$. \\ Harald Schuh $^{3}$
}

Received: 20 October 2015 / Accepted: 23 July 2016

(C) Springer-Verlag Berlin Heidelberg 2016

\begin{abstract}
Precise transformation between the celestial reference frames (CRF) and terrestrial reference frames (TRF) is needed for many purposes in Earth and space sciences. According to the Global Geodetic Observing System (GGOS) recommendations, the accuracy of positions and stability of reference frames should reach $1 \mathrm{~mm}$ and $0.1 \mathrm{~mm}$ year $^{-1}$, and thus, the Earth Orientation Parameters (EOP) should be estimated with similar accuracy. Different realizations of TRFs, based on the combination of solutions from four different space geodetic techniques, and CRFs, based on a single technique only (VLBI, Very Long Baseline Interferometry), might cause a slow degradation of the consistency among EOP, CRFs, and TRFs (e.g., because of differences in geometry, orientation and scale) and a misalignment of the current conventional EOP series, IERS 08 C04. We empirically assess the consistency among the conventional reference frames and EOP by analyzing the record of VLBI sessions since 1990 with varied settings to reflect the impact of changing frames or other processing strategies on the EOP estimates. Our tests show that the EOP estimates are insensitive to CRF changes, but sensitive to TRF variations and unmodeled geophysical signals at the GGOS level. The differences between the conventional IERS $08 \mathrm{C} 04$ and other EOP series computed with distinct TRF settings exhibit biases and even non-negligible trends in the cases where no differential rotations should appear, e.g., a drift of about 20
\end{abstract}

Santiago Belda santiago.belda@ua.es

1 Department of Applied Mathematics, EPS, University of Alicante, 03080 Alicante, Spain

2 Departamento de Matemática Aplicada, University of Alicante, Alicante, Spain

3 Helmholtz Centre Potsdam, GFZ German Research Centre for Geosciences, Potsdam, Germany $\mu$ as year ${ }^{-1}$ in $y_{\text {pol }}$ when the VLBI-only frame VTRF2008 is used. Likewise, different strategies on station position modeling originate scatters larger than $150 \mu$ as in the terrestrial pole coordinates.

Keywords Earth orientation parameters - Reference systems $\cdot$ Reference frames $\cdot$ VLBI

\section{Introduction}

Assessing the actual accuracy of the earth orientation parameters (EOP) is still an open and timely question, into which we need more insight in view of the demanding requirements of accuracy and stability pursued at present by, e.g., GGOS, the Global Geodetic Observing System of the International Association of Geodesy (IAG)_Plag and Pearlman (2009). GGOS goals are $1 \mathrm{~mm}$ in accuracy and $0.1 \mathrm{~mm} /$ year in stability of the reference frames; those values, when measured on the Earth surface, correspond, respectively, to just above $30 \mu$ as and $3 \mu$ as/year in terms of angles from the Earth's centre, or $2 \mu \mathrm{s}$ and $0.2 \mu \mathrm{s} / \mathrm{year}$ in time units, and they were adopted by the IAU/IAG Joint Working Group on Theory of Earth Rotation (Ferrándiz and Gross 2014). Operational EOP are provided for worldwide use by the Earth Orientation Centre (EOC) of the International Earth Rotation and Reference System Service (IERS); IERS also hosts for the product centers: the conventional International Celestial and Terrestrial Reference Frames (ICRF and ITRF, respectively). According to the IERS Conventions (2010) (Petit and Luzum 2010), the conventional daily EOP are currently realized by the time series IERS $08 \mathrm{C} 04$ that links the conventional realization of the ICRS, currently ICRF2 (Fey et al. 2015) to the conventional realization of the ITRS denoted ITRF2008 (Altamimi et al. 2011). 
The computation of the ITRF depends on a complex process, in which the solutions produced by the four main space geodetic techniques and by various analysis centers (AC) are combined. Regarding input data, it is assumed that each technique refers to its own reference system and, furthermore, each coordinate epoch refers to a separate reference system (Altamimi et al. 2011). The stacking is performed in two steps, the first is applied to data from each single technique separately and the second brings the former results together to derive a common ITRF. The concurrence of all those factors is a source of intricacies and makes difficult the assessment of the actual accuracy of the EOP. In any case, the solutions for EOP and ITRF are obtained, so that they provide optimal consistency among them, according to certain optimality criteria that involve the least-squares minimization of unknown parameters or apparent coordinate variations as described in detail, e.g., in Altamimi and Dermanis (2012). However, whereas the nature of an ITRF compels it to last for some years and be "frozen" during a certain period before the release of the next reference frame, the EOP must be provided on a more continuous basis. The IERS 08 C04 conventional EOP series are also produced under a combination process that consists of several steps and gathers data from all techniques. It is detailed in Bizouard and Gambis (2011). This combination process is unconnected to the ITRF combination, in the sense that the EOP solution is not forced to coincide with the solution computed along with the ITRF in their common time span, but it is computed from the technique-wise EOP solutions imposing certain constraints, as for instance, the absence of trends w.r.t. the ITRF2008. Of course, neither the accuracy nor the consistency between the EOP determined from data beyond the time interval used in the realization of the reference frames, and those frames themselves can be ensured a priori and must be estimated a posteriori. It is clear than the accuracy of the resulting EOP solution cannot surpass that of the implied frames, but could be worse. In this complex situation, accuracy is usually estimated in terms of formal errors, uncertainties, or repeatability, and the assessment of the actual (not the assumed) accuracy of the current conventional EOP becomes a cumbersome issue, though tightly linked to the level of consistency between the IERS 08 C04 series, ITRF2008, and ICRF2.

The objective of this article is to investigate the issue of the mutual consistency of the time series IERS 08 C04 together with ITRF2008 and ICRF2, not restricted to the time interval used for the frame building. The procedure relies on performing suitable analysis of observational data. We follow the standard ideas used in the validation of empirical models, which requires analyzing the residuals between models and observations, irrespective of the simplicity or complexity of the model. It seems reasonable that the first step should be the analysis of VLBI data, since VLBI is the only technique capable of providing operative solutions for the whole set of
EOP. The analysis could provide more insight not only into accuracy or consistency issues, but also into the features of VLBI solutions compared with combined solutions and into the current limits of model improvement.

Our analyses comprise all the VLBI sessions between 1990 and 2013. The EOP are derived in the form of time series similar to the conventional ones, each one corresponding to distinct changes in the processing settings which are explained in detail in Sects. 2 and 3. Section 3 comprises several subsections, each one covering a test problem empirically. In the first subsection, we present the results of an experiment designed to assess the effect of unrestricted, unmodeled geophysical signals on the EOP series. Next, the sensitivity of the VLBI EOP solutions to the change of the a priori EOP series is addressed. In Sects. 3.3 and 3.4, we test some TRF and CRF realizations (distinct from those used in the IERS $08 \mathrm{C} 04$ derivation) to study their impact on the EOP, especially in the long-term, paying attention to the appearance of biases and especially trends among the different EOP series, which would suggest the emergence of differential rotations. The last Sect. 3.5 aims at discerning to which extent the behaviour found in Sect. 3.3 can be attributed to differential orientations of the TRFs. Finally, in Sect. 4, the main points of the former individual experiments are summarized and discussed, and the conclusions are drawn.

The results in this article are an extension and continuation of our previous results contained in two conference papers by Heinkelmann et al. (2014b, 2015). Those papers introduce the basic ideas and methodology in a concise way and emphasize on the interpretation of the results and the discussion of the consistency among frames rather than on EOP. In those previous analyses, all the VLBI sessions since 1984 were accounted for. Here, we decided to remove them from the analysis as recommended by different authors (Malkin 2013b; Chao and Hsieh 2015), in view of the small magnitude of the effects found in the first analysis and the inaccuracy of data in the earlier years. In this case, the modification of the analysis period does not produce substantial qualitative changes, apart from the differences in the numerical results displayed in Table 3 here and Table 1 in Heinkelmann et al. (2015). Sections 3.2 and 3.5 do not have a counterpart in the precedent studies.

\section{Data analysis}

The consistency issues are assessed by performing different VLBI data analyses, which are extended to 2912 sessions ranging from 1990-01-18 until 2013-12-31 (GFZ VLBI contribution to ITRF2013; Heinkelmann et al. 2014a); the initial years until 1990 have been excluded from the analysis due to the lower quality of the VLBI data. The GFZ version 
of the Vienna VLBI software (VieVS, Böhm et al. 2012), VieVS@GFZ, was utilized, with the following common processing options: for each EOP, one offset per day with respect to a selected a priori series (usually IERS 08 C04) was estimated for each VLBI session. For modeling, the tropospheric delays we used the Vienna mapping functions (VMF1, Böhm et al. 2006), and we estimated the zenith wet delays and the tropospheric gradients as piece-wise linear functions with $1 \mathrm{~h}$ and $6 \mathrm{~h}$ interval lengths, respectively. The station clock offsets were estimated as piece-wise linear functions with $1 \mathrm{~h}$ interval lengths, plus quadratic terms (Nilsson et al. 2014). After single-session adjustments, we discarded about 50 VLBI sessions with a posteriori sigma of unit weight larger than 3.

Other processing options depend on the different analyses that have been performed and will be detailed in the corresponding sections. For instance, when we intended to determine the effect of a specific TRF or CRF on the EOP, we fixed the station and source coordinates on their catalogue values. Thus, various EOP series were determined using different celestial (Table 1) and terrestrial (Table 2) reference frames for computing each solution and varying the a priori EOP series (IERS 08 C04, USNO finals, and IAU 2000/2006 precession-nutation models). When the aforementioned products are evaluated using VLBI data only, it should be remarked that the assessment would show the (in)consistency among the products with respect to VLBI data, but the results should not be extrapolated to other techniques.

To compare the different pairs of EOP time series estimates, we calculated the Weighted Mean (WM) of the

Table 1 Different CRFs used in this study

\begin{tabular}{lll}
\hline CRF & References & Comment \\
\hline ICRF-Ext.2 & Ma et al. (1998) & $\begin{array}{c}\text { Orientation is based on } \\
\text { data until 1995.5 }\end{array}$ \\
& Fey et al. (2004) & \\
ICRF2 & Ma (2009) & Data until (2009) \\
& Fey et al. (2015) & \\
\hline
\end{tabular}

differences and the Weighted Root Mean Square (WRMS) differences between each of them, by means of the following formulae (Nilsson et al. 2014), where sub-indices eop1 and eop2 denote the individual solution:

$$
\begin{gathered}
\mathrm{WM}=\frac{\sum_{i=1}^{N} \frac{\tilde{x}_{\mathrm{eop} 1, i}-\tilde{x}_{\mathrm{eop} 2, i}}{\sigma_{\mathrm{eop} 1, i}^{2}+\sigma_{\mathrm{eop}, i}^{2}}}{\sum_{i=1}^{N} \frac{1}{\sigma_{\mathrm{eop} 1, i}^{2}+\sigma_{\mathrm{eop} 2, i}^{2}}} \\
\mathrm{WRMS}=\sqrt{\frac{\sum_{i=1}^{N} \frac{\left(\tilde{x}_{\mathrm{eop} 1, i}-\tilde{x}_{\mathrm{eop} 2, i}-\mathrm{WM}\right)^{2}}{\sigma_{\mathrm{eop} 1, i}^{2}+\sigma_{\mathrm{eop} 2, i}^{2}}}{\sum_{i=1}^{N} \frac{1}{\sigma_{\mathrm{eop} 1, i}^{2}+\sigma_{\mathrm{eop} 2, i}^{2}}}}
\end{gathered}
$$

where, $\tilde{x}$ denote the estimates of EOP values from the VLBI analysis using the different settings, $N$ their number and $\sigma$ indicate their respective formal uncertainties. Moreover, when analyzing the residuals between a pair of different EOP solutions, a linear trend was computed, composed of a shift (referred to epoch J2000.0) and a linear drift calculated by Least Squares (LS) or Weighted Least Squares (WLS), where the error of fits was assessed by the weighted root mean square (denoted by WRMS).

Suitable statistical tests were also applied to ensure that the results are statistically significant at the 0.05 level $(\alpha)$ before drawing conclusions. Since the series are normally distributed, the WM values were analyzed by $t$ test and the WRMS values by $F$ test.

\section{Results}

\subsection{Unmodeled geophysical signals}

The first analysis is concerned with the effect of unmodeled geophysical signals affecting the position of VLBI stations. Notice that unmodeled is used in the proper sense of a component of a signal not accounted in a given model adopted in the processing strategy, it should not be understood necessarily as a deficiency of a conventional model. Let us recall that the determined station coordinates do not have a simple dependency on geophysical signals, since several models rec-
Table 2 Different TRFs used in this study

\begin{tabular}{lll}
\hline TRF & Reference & Comment \\
\hline ITRF2000 & Altamimi et al. (2002) & Data until 2000 \\
ITRF2005 & Altamimi et al. (2007) & Data until 2005 \\
ITRF2008 & Altamimi et al. (2011) & Data until 2008 \\
VTRF2008 & Böckmann et al. (2010) & $\begin{array}{c}\text { Data until 2008, VLBI-only frame, contains the same VLBI } \\
\text { data as it was provided to ITRF2008 and DTRF2008 }\end{array}$ \\
DTRF2008 & Seitz et al. (2012) & $\begin{array}{c}\text { Data until 2008, contains the same data as ITRF2008, but } \\
\text { using a different combination approach }\end{array}$
\end{tabular}


ommended in the IERS Conventions (i.e., solid Earth tides, oceanic, and atmospheric tidal loading) are applied as a priori models in various data analyses, including the TRF estimation. However, other geophysical effects (i.e., non-tidal atmosphere, non-tidal ocean, and hydrological loading) are not recommended for the conventional analyses; their effect on the results is accumulated together with the inaccuracies of the considered a priori models. Besides, the adopted model for the station coordinates is linear, made up of a position and a constant velocity. The consequence of all that is that the appearance of unmodeled geophysical signals may propagate into inaccuracies of the EOP.

That possibility was clearly confirmed in a recent paper by Krásná et al. (2015). They estimated three different VLBI solutions to evaluate the impact of the unmodeled seasonal signals in the station displacement on the CRF and EOP. In the first solution, the seasonal displacement was omitted (reference signal). In the second alternative harmonic corrections, composed of annual and semi-annual constituents, they were computed for a number of stations and used to improve the model of their displacements. In addition, in the third option, the seasonal displacement was modeled with a mean annual model, which had been described and applied by Tesmer et al. (2009). With this analysis, Krásná et al. (2015) unveiled the existence of differences of several tens of $\mu$ as into the ERP determinations, as well as large drifts (1.82 $\mu$ as year ${ }^{-1}$ in $y_{\text {pol }}$ and $-0.10 \mu$ s year ${ }^{-1}$ in dUT1) when VieTRF13b and VieCRF13b (Krásná et al. 2014) were used as a priori reference frames together with the harmonic model. These results agree with the previous studies performed by Ding et al. (2005), Tesmer et al. (2009), Malkin (2013a), and Eriksson and MacMillan (2014), who found unmodeled annual and semi-annual displacements in the station horizontal coordinates. Let us recall that the current conventional standards for the station motions only account for a constant velocity term-although they will be extended in the next TRF realization, ITRF2014. For these model limitations and to avoid damaging effects, fixing station coordinates to their a priori values is not recommended by the scientific community, in general, and in particular when estimating EOP.

In this test, we neither determine nor apply seasonal components to correct the station positions, since we aim at assessing the uncertainty and stability of the current conventional products (ITRF2008, ICRF2, and IERS 08 C04), attributable to any kind of unmodeled geophysical signal (seasonal or not) affecting the regularized station coordinates reported in the ITRF2008 catalogue. We proceed by estimating the EOP through two different approaches:

(a) FIXED ITRF2008 coordinates (unmodeled geophysical signals propagate into EOP). (b) FREE ITRF2008 coordinates (unmodeled geophysical signals cause adjustments of station coordinates). This means that the positions and velocities of all station coordinates were estimated by imposing no-net-translation and no-net-rotation conditions with respect to ITRF2008.

For numerical assessment, we compare the resulting EOP series (approach a vs. b) to quantify how important the effects of the unmodeled geophysical signals are. The first global indicators of the differences are provided by the mean and the dispersion of the differences of the series computed for each EOP. Table 3 displays the WM and WRMS differences between the two solutions and shows the shifts and drifts of the EOP differences w.r.t. IERS $08 \mathrm{C0} 4$ and the correlations among the two approaches. Fixing station positions to their nominal values in the ITRF2008 catalogue causes no statistically significant ( $p$ value $>0.05$ ) WM differences of all EOP; however, it generates noticeable scatter between both solutions, that reaches about 144, 164, and $5.9 \mu$ s for the differences in $x_{\text {pol }}, y_{\text {pol }}$, and dUT1, respectively.

The celestial pole offsets (CPO) are insignificantly affected by unmodeled signals: the correlation coefficients between solutions following approach a and approach $b$ are very large (0.95 and 0.94), and their WRMS are not significant ( $p$ value $>0.05$ ) affected by the approach (Table 2). However, we notice a small shift of $15 \mu$ as and a drift at the level of 3 $\mu$ as/year of the $Y$ component of the CPO in both approaches; that value is at the limit of the GGOS stability goal. That drift is nearly the same found in our previous work, including the VLBI sessions since 1984, but in that case, there also appeared a shift of dUT1 with a magnitude of $5.7 \mu \mathrm{s}$, much larger than the shifts displayed in Table 3, at the level of 4.5 $\mu \mathrm{s}$ for dUT1. The $4.5 \mu \mathrm{s}$ shift for dUT1 is significantly larger than the GGOS goal. If the results are compared with Table 1 in Heinkelmann et al. (2015), which covers 1984-2013, there is a pattern common to all the EOPs, namely very close drifts and different biases.

Concerning Table 3 it is important to note that the different handling of the TRF station coordinates (fixed and free approach) results in a strong decrease of the correlation between each series of Earth Rotation Parameters (ERP) strategy as a consequence of the neglected signals, which happens to be close to $50 \%$ in the case of the pole coordinates and is consistent with the large values of the WRMS shown in the last column of Table 3, with an average near 150 $\mu$ as. That large scatter is about five times the GGOS accuracy target, what confirms that the modeling of the station position is one of the key problems to improve the EOP repeatability. Besides, the WRMS (weighted root mean square after subtracting the linear component of the difference) of the ERP especially increase from the fixed to the free approach; our explanation for this fact is that the IERS 08 C04 EOP are consistent with the linear station model of ITRF2008, and 
consequently, if station coordinates differ from their catalogue value, the EOP scatter will increase. Figure 1 displays the ERP differences w.r.t. IERS $08 \mathrm{C0} 4$ for the fixed and free approaches to help to graphically decipher the time scales present in the large differences and reduced correlations. As noted by Krásná et al. (2015), the main discrepancies present a dominant annual pattern. However, these differences cannot be only modeled with annual and semi-annual constituents, since they are composed of more complex signals. This fact can be seen in the periodograms of the ERP differences between both approaches (Fig. 2). Regarding the pole coordinates, the maximal spectral power is located near the 1 year period, heterogeneous patterns of higher frequencies being visible too. Their provenance is unclear, but some of them may have actual physical origin. In any case, we have fitted annual and semi-annual harmonic constituents to each EOP and show the results also in Table 3, to compare them to Krasna's et al. results. The orders of magnitude are similar, which provides additional evidence on the consistency level. Our values are closer to their S3-S1 difference in Table 8, which do not use harmonic models for the seasonal station positions but non-linear annual means. That fact suggest that the use of a harmonic model for the station position variations may have more significant impact on EOP than using smoother or no models, although more insight in the issue is needed to draw a conclusion.

\subsection{Different a priori EOP series}

In an ideal case, the estimated values of the EOP should be the same independent of the a priori values. However, the highly accurate estimation of the full set of EOP is not simple from either a mathematical or physical perspective, and the possibility of having effects derived from the choice of the initial solution should be investigated. In this test, several a priori EOP series were used to estimate the EOP by VLBI fixing the reference frames to the current conventional ITRF2008 and ICRF2. First, VLBI time series were determined using a priori EOP from IERS $08 \mathrm{C0} 4$ (case 1), second, the a priori ERP and Celestial Pole Coordinates were taken from IERS 08 C04 and from the IAU 2006/2000A precessionnutation model, respectively (case 2), and finally, the USNO Finals time series were used as a priori EOP values (case 3 ). The comparison shows no significant $(p$ value $>0.05)$ EOP WM differences between the IERS 08 C04 and USNO Finals approaches (cases 3 vs 1), whereas their repeatabilities (measured by the WRMS) are close to $40 \mu$ as in all the EOP with the exception of dUT1 that is around $5.3 \mu$ s, i.e., more than 2.5 times the corresponding GGOS accuracy of $2 \mu \mathrm{s}$ (Fig. 3). EOP residuals reveal almost negligible shifts and drifts (Table 4). Therefore, VLBI-determined EOP using either IERS $08 \mathrm{C} 04$ or USNO Finals as a priori values are in a very good agreement (correlation about 0.93 ). 

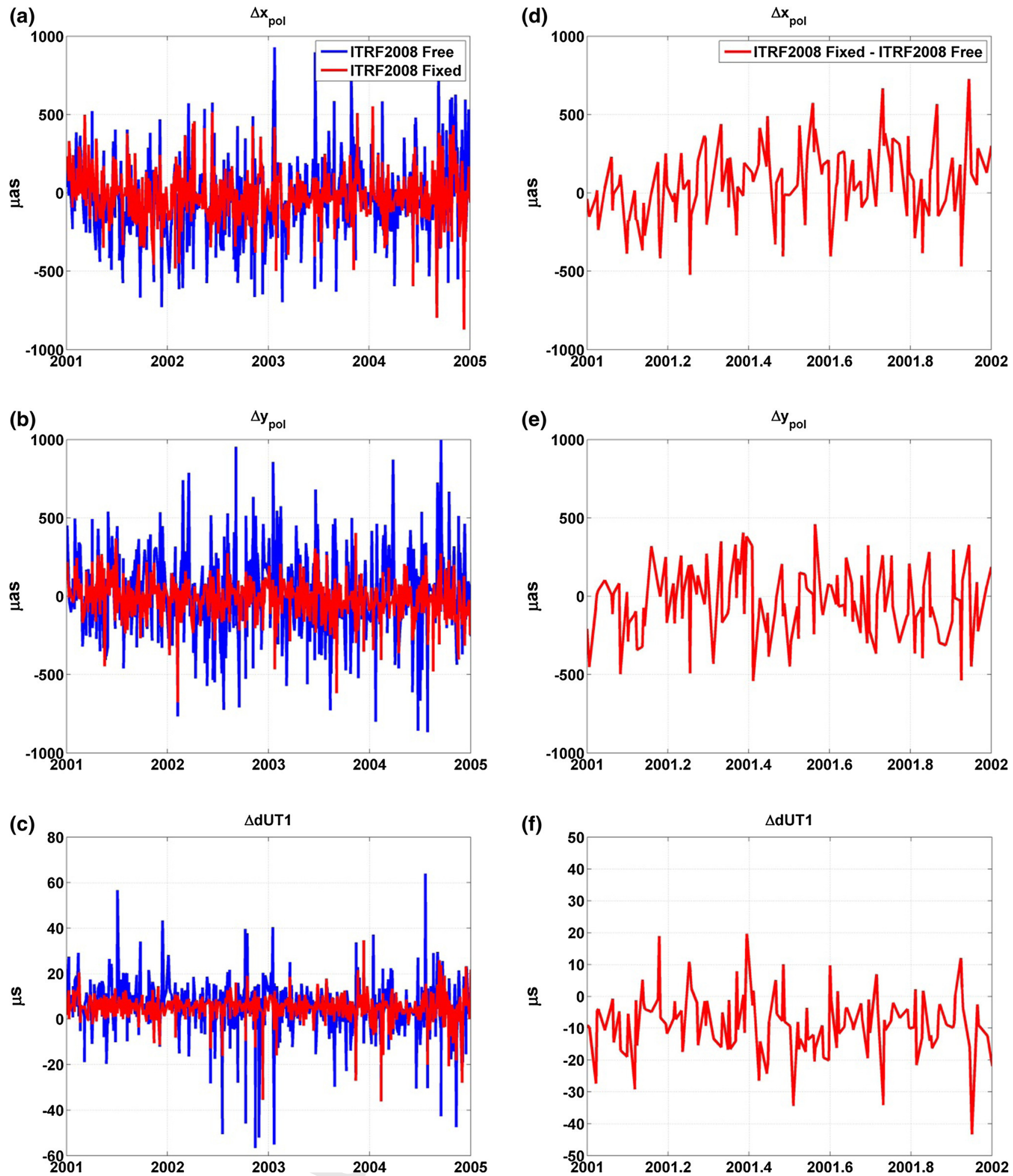

Fig. 1 ERP residuals w.r.t. IERS 08 C04 between solutions using fixed (red line) and free (blue line) ITRF2008 coordinates observed from 2001 to 2005 (left column). Differences between both solutions zoomed-in from 2001 to 2002 (right column). Units $\mu$ as or $\mu$ s for dUT1

The interpretation of the differences between the cases 1 and 2 is not so simple (Fig. 3). First, the WM differences of the polar motion (PM) parameters and dUT1 are insignificant ( $p$ value $>0.05$ ), as it could be expected, since the a priori values for the three ERP were not changed. It is important to remark that the WM results correlate strongly with the shifts listed in 

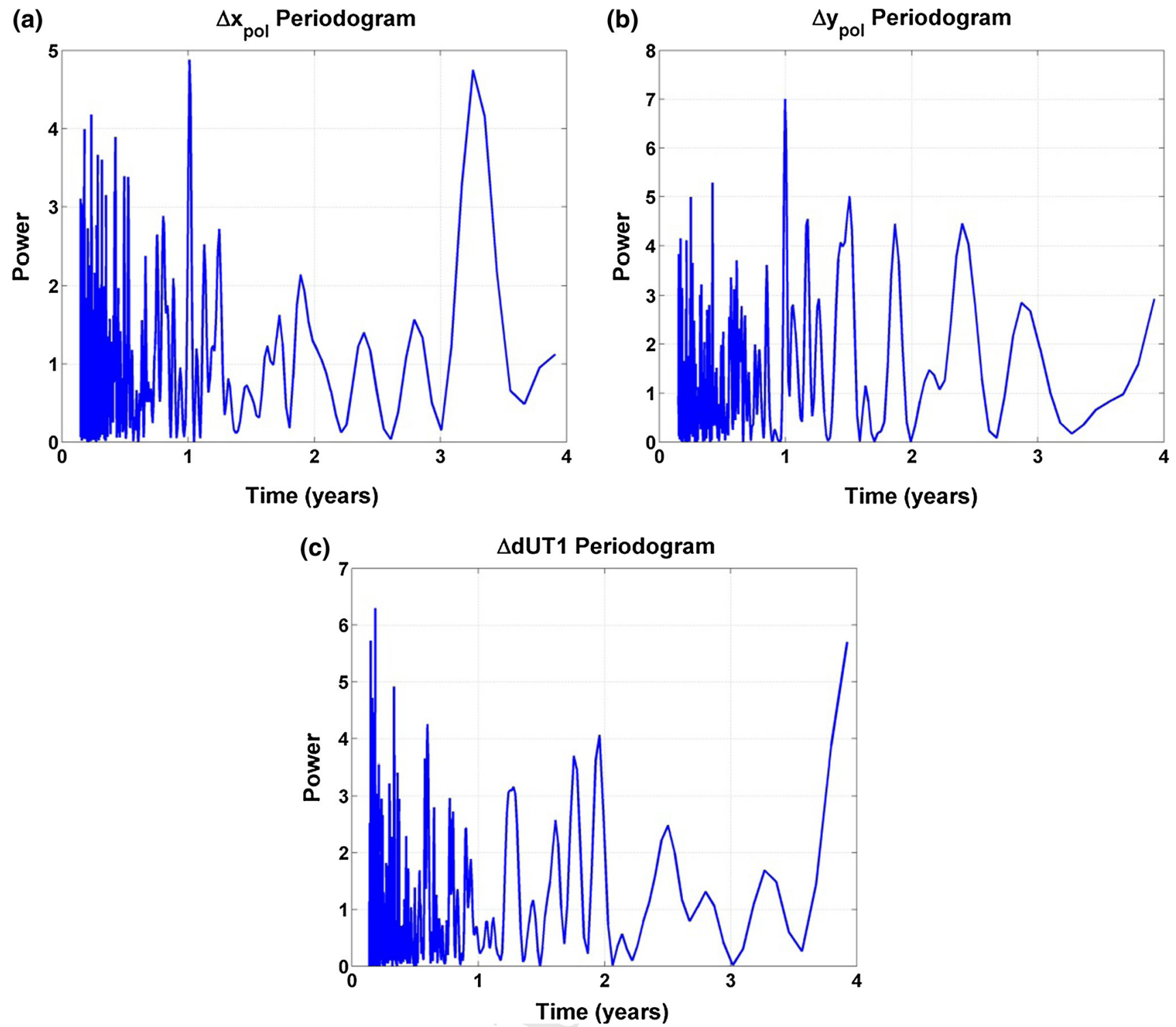

Fig. 2 Periodograms of the differences between ERP estimated by different approaches: fixed and free ITRF2008 coordinates. A priori EOP series: IERS 08 C04

Table 4. As for the WRMS values, in contrast to the precedent case, they are about $20 \mu$ as for both PM components and smaller for dUT1. The differences between the CPO are much more significant ( $p$ value $<0.05$ ), as expected, since they correspond roughly to the deviation between the conventional nutation theory and the operational solution. It is known that the IAU-adopted precession/nutation model, currently IAU 2006/2000A (Dehant 2002; Hilton et al. 2006), contains only the easier to predict, forced astronomical effects, and thus, the Free Core Nutation (FCN) is not included. This has a powerful impact on the residuals-apart from the need of some additional corrections. Besides WRMS over $160 \mu$ as, we detect statistically significant WM differences $(-55 \mu$ as in $\mathrm{d} X$ and $93 \mu$ as in $\mathrm{d} Y)$ and large shifts $(-39.8 \mu$ as in $\mathrm{d} X$ and $94.2 \mu$ as in $\mathrm{d} Y$ ) (Table 4 ) of the CPO between the IERS 08 C04 and IAU 2006/2000A approaches with a significance level 0.05, showing considerable scattering (WRMS of about $160 \mu$ as). The importance of using a good FCN model to get smaller residuals is well known. Nowadays, several empirical models are available with high temporal resolution and accuracy (Lambert 2007; Malkin 2010, 2013b; Krásná et al. 2013; Belda et al. 2016). We modify the case 2 by adding to IAU 2006/2000A the model determined recently by Belda et al. (2016), which was fitted to VLBI data using a sliding window length of 400 days displaced 1 day and a constant period of -431.18 sidereal days for the signal, so that we can remove the FCN oscillations that appear in case 2 (Fig. 4). To study the remaining residuals $([\Delta X, \Delta Y]=\mathrm{CPO}-\mathrm{FCN})$, the 

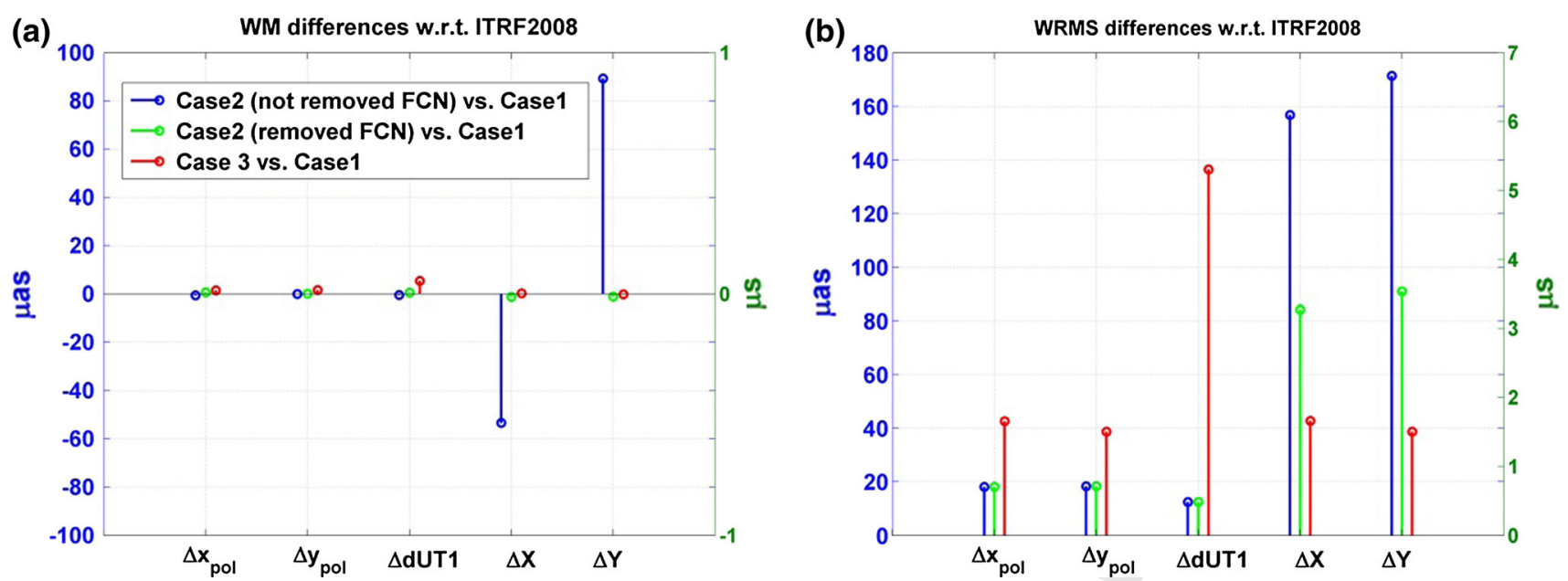

Fig. 3 WM and WRMS differences between EOP estimated with different a priori EOP. Case 1 EOP $\rightarrow$ IERS 08 C04. Case 2 ERP $\rightarrow$ IERS 08 C04 and $X, Y \rightarrow$ IAU 2006/2000A. Case 3 EOP $\rightarrow$ USNO Finals. Units: $\mu$ as (left side) or $\mu$ s (right side) for dUT1

Table 4 EOP Differences ( $\mu$ as or $\mu$ s for dUT1) between solutions using different a priori EOP series

\begin{tabular}{|c|c|c|c|c|c|c|}
\hline \multirow[t]{2}{*}{ EOP } & \multicolumn{3}{|c|}{ Case 2 vs. case 1} & \multicolumn{3}{|c|}{ Case 3 vs. case 1} \\
\hline & Shift & Drift & WRMS & Shift & Drift & WRMS \\
\hline$\Delta x_{\mathrm{pol}}(\mu \mathrm{as})$ & $-0.9 \pm 1.1$ & $0.0 \pm 0.1$ & 18.1 & $-0.9 \pm 2.7$ & $-0.1 \pm 0.2$ & 42.6 \\
\hline$\Delta y_{\mathrm{pol}}(\mu \mathrm{as})$ & $-0.7 \pm 1.1$ & $0.1 \pm 0.1$ & 18.4 & $-2.4 \pm 2.3$ & $0.2 \pm 0.1$ & 38.7 \\
\hline$\Delta \mathrm{dUT} 1(\mu \mathrm{s})$ & $0.0 \pm 0.03$ & $0.0 \pm 0.01$ & 0.5 & $0.0 \pm 0.02$ & $0.0 \pm 0.02$ & 5.3 \\
\hline$\Delta X(\mu$ as $)$ & $-39.8 \pm 8.9$ & $-2.5 \pm 0.5$ & 156.2 & $0.7 \pm 2.4$ & $-0.2 \pm 0.1$ & 42.8 \\
\hline$\Delta Y(\mu$ as $)$ & $94.2 \pm 9.9$ & $-0.9 \pm 0.6$ & 171.3 & $-0.3 \pm 2.2$ & $0.1 \pm 0.1$ & 38.7 \\
\hline
\end{tabular}

Shift (referred at epoch J2000.0) and linear trends $\left(\right.$ year $^{-1}$ ) are estimated by WLS. The WRMS are computed after subtracting the linear component of the difference

Case 1 EOP $\rightarrow$ IERS 08 C04. Case 2 ERP $\rightarrow$ IERS 08 C04 and $X, Y \rightarrow$ IAU 2006/2000A. Case $3 \mathrm{EOP} \rightarrow$ USNO Finals (a)

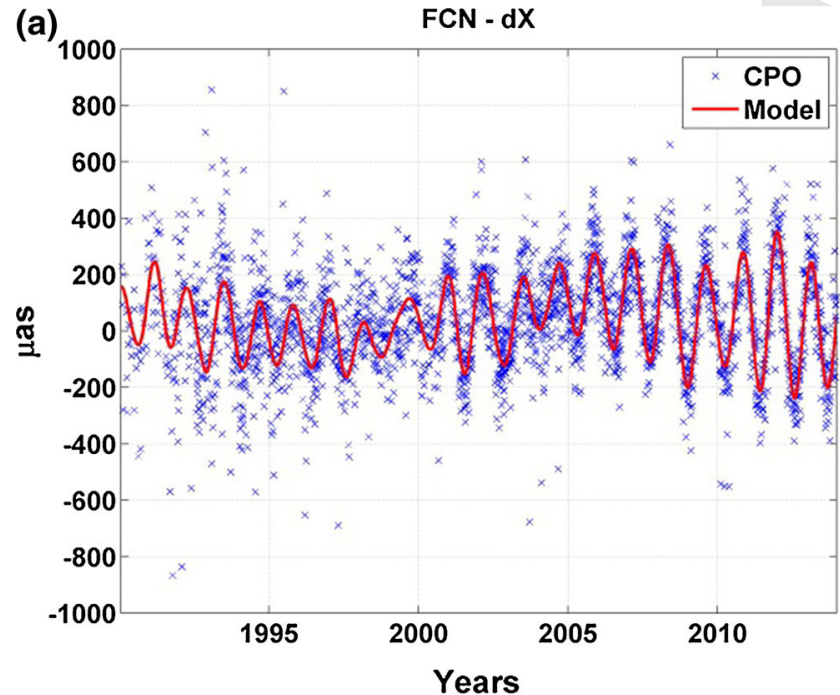

(b)

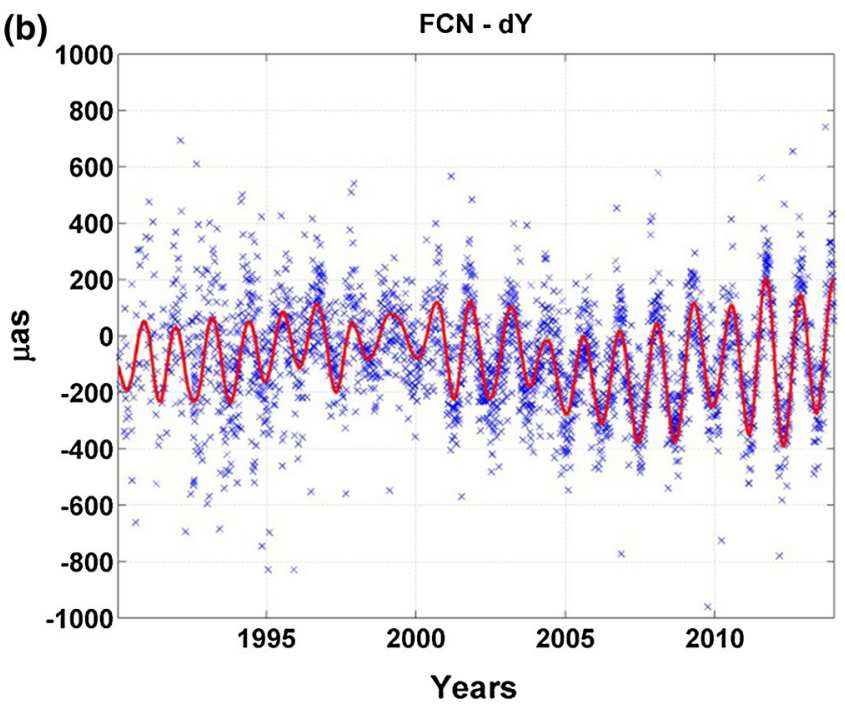

Fig. 4 Blue dots CPO estimated from IERS 08 C04 as a priori ERP and the Celestial Intermediate Pole (CIP) coordinates from the IAU 2006/2000A precession/nutation theory (blue dots). Red line Empirical Free Core Nutation (FCN) model plus the low-frequency part of the signal. Units $\mu$ as 
WM and WRMS differences were estimated between case 1 and case 2 (with and without modification), once the FCN was deleted (Fig. 3) (red line). It is noteworthy to mention that the usage of the aforementioned model causes insubstantial WM differences with a WRMS of about $80 \mu$ as in the CPO; that reduction of the scatter by almost a half is remarkable and the remaining variance seems to be attributable to the limitations of the theory and the models in terms of unmodeled contributions.

\subsection{Terrestrial reference frames}

The impact of using different TRFs to compute EOP solutions is assessed by fixing the station coordinates to their a priori values taken from the respective catalogues. Fixing the station positions entails somehow a deformation of the actual network, since some unmodeled geophysical signals still remain in every TRF apart from other possible sources of uncertainty; however, this procedure is necessary here to determine the differences between the investigated catalogues: if the coordinates would not be fixed on catalogue values for this purpose, the VLBI data adjustment would change the coordinates and we would not be able to assess the consistency. Therefore, several EOP series were estimated using the five terrestrial reference frames given in Table 5; in all the cases, we took IERS $08 \mathrm{C} 04$ as a priori EOP values and fixed the radio source coordinates from ICRF2. Notice that the estimates for each TRF (Fig. 5) are obtained using the same VLBI sessions, holding the $3 \sigma$ eligibility criterion for all the implied frames, to achieve the maximum coherence degree in the comparison.

The shift and drift of the EOP offsets provided by VieVS using the different TRFs w.r.t. IERS $08 \mathrm{C} 04$ were computed (Table 5) to compare the residuals associated with each TRF with the ITRF2008 case. One of the most significant results for $x_{\text {pol }}$ corresponds to the ITRF2000 case, exhibiting considerable shift and drift $(-123.9 \mu$ as and $-16.8 \mu$ as year $^{-1}$, respectively), followed in magnitude by its high drift in ITRF2005 (-9.0 $\mu$ as year $\left.{ }^{-1}\right)$. Examining the case of ITRF2005 is interesting, since it corresponds to the most recent change of ITRF and EOP releases. It is known that the seven-parameter rotation and rotation rates relating ITRF2008 and ITR2005 vanish with the reported formal errors being of $8 \mu$ as. However, according to Sect. 3.5.1 of the IERS Annual Report 2011 (Dick 2013), the IERS 05 C04 series referred to ITRF2005 were re-aligned recognizing the following biases, most of them given without formal errors: "negligible" in $x_{\mathrm{pol}},-50 \pm 25 \mu$ as in $y_{\mathrm{pol}}, 2 \mu \mathrm{s}$ in $\mathrm{dUT} 1$, and 1 and $17 \mu$ as in $\mathrm{d} X, \mathrm{~d} Y$, respectively. Following the same order and same units (for ITRF2005 in Table 5), we find biases of values $-25,27,-1,-2$, and 16 , respectively, but the remarkable novelty with respect to the presumed relative orientation of the experiment results is the appearance

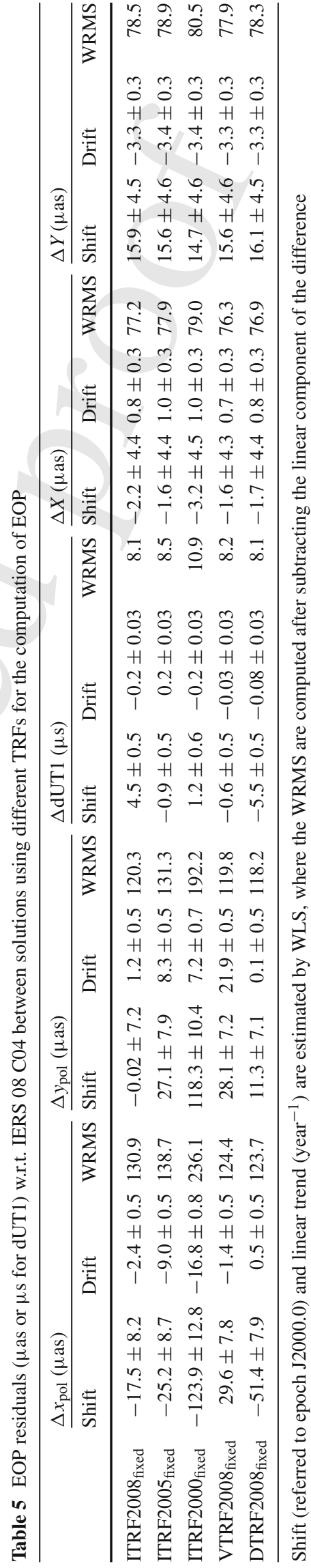



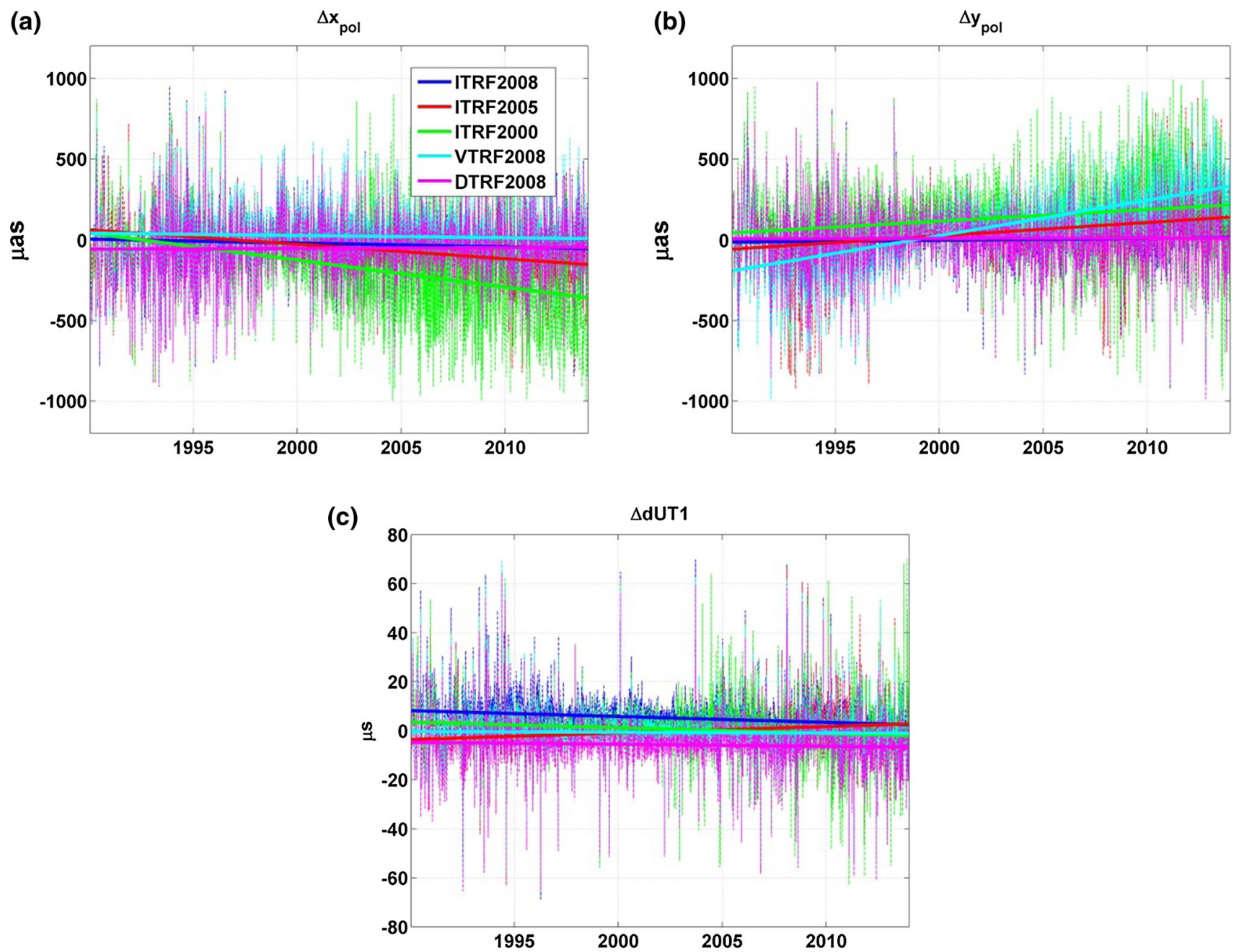

(d)

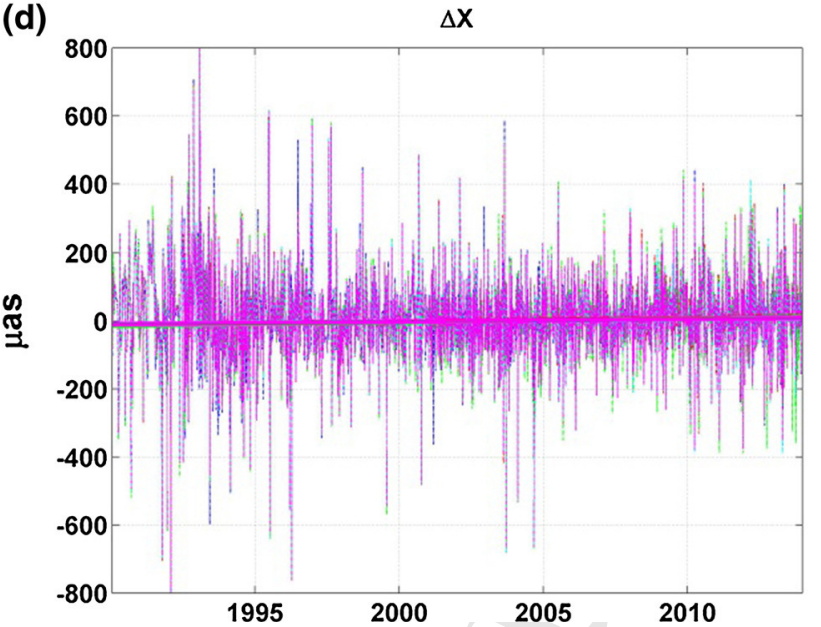

(e)

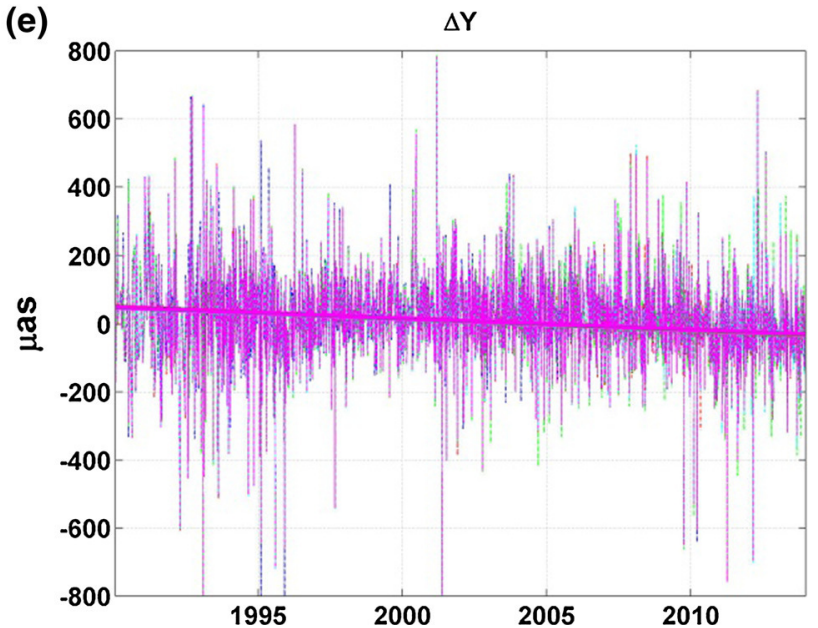

Fig. 5 EOP differences w.r.t. IERS 08 C04 between solutions using different terrestrial reference frames (blue ITRF2008, red ITRF2005, cyan VTRF2008, magenta DTRF2008 and green ITRF2000). Straight lines represent the corresponding linear trends. Units $\mu$ as or $\mu$ s for dUT1

of non-negligible drifts, two of them reaching the 8-9 $\mu$ as year ${ }^{-1}$ magnitude. That issue is addressed in Sect. 3.5 from a different perspective to get more insight.
Relevant weakness for the $y_{\text {pol }}$ parameter appears in ${ }_{464}$ VTRF2008 and ITRF2000, with a trend bigger than $20 \mu$ as $\quad 465$ year $^{-1}$ and an important shift of $118.3 \mu$ as, respectively. Con- $\quad 466$ 
cerning DTRF2008 and the ERP, the maximal shift occurs in dUT1, exceeding $-5.5 \mu \mathrm{s}$; the shift of $x_{\text {pol }}$ reaches $-51 \mu$ as. Finally, trends and biases of the CPOs are close in all the cases and smaller than those of the ERPs. The maximum scatter and minimum scatter (WRMS after subtracting the linear component of the difference w.r.t. IERS $08 \mathrm{C} 04$ series) of the EOP correspond to ITRF2000 and DTRF2008, respectively.

ITRF2000 presents large WM and WRMS in $x_{\mathrm{pol}}, y_{\mathrm{pol}}$, and dUT1. Further interesting results are found for the VTRF2008 case, with large WM (-133.8 $\mu$ as), WRMS (113.0 $\mu \mathrm{as})$, for $y_{\mathrm{pol}}$; that seems to be due to the contrast between multi-technique (ITRF2008) vs single-technique (VTRF2008) approaches. DTRF2008 shows large WM (10.5 $\mu \mathrm{s})$ for dUT1, which is remarkable, because both ITRF2008 and DTRF2008 are based on the same input data and accordingly, they only differ in the weighting of the techniques and the local ties among each other and in the datum definition. Celestial pole coordinates in all the cases do not show noticeable systematic effects, with small WM and WRMS (5 and $10 \mu$ as, respectively). It is evident that the CPO are insensitive to TRF changes unlike the ERP, within the accuracy and stability limits set by GGOS.

\subsection{Celestial reference frames}

Other EOP solution series were calculated using two different Celestial Reference Frames (ICRF2 and ICRF-Ext.2, Table 1) to study the sensitivity of VLBI EOP to the a priori CRF. In this part, the conventional terrestrial reference frame (ITRF2008 fixed on its a priori values) was used for the VLBI analysis, together with the IERS $08 \mathrm{C0} 4$ as a priori EOP. As in the previous sections, the difference between both approaches is assessed by means of WM and WRMS. According to our estimates, the impact of using two different ICRFs on EOP is about at the level of stability of the ICRF2 axes relative to ICRF-Ext.2 (10 $\mu$ as) with WRMS EOP differences of about $40 \mu$ as (Table 6). The fact that errors in source positions affect EOP in a much lesser extent than errors in station position is not unexpected at all, since in ordinary VLBI sessions, the number of observed sources is much larger than the number of participating stations. It also empirically confirms that the statistics given for the ICRF2 are correct and it proves that the additional including of about 15 years of VLBI observations (comparing ICRF-Ext. 2 and ICRF2) does not lead to systematic rotations of the ICRF. Shifts and drifts of the differences between both ICRF solutions (reported in Table 6) present analogous results for both studies, where the largest WRMS of the linear regression can be found on the EOP corresponding to ICRF-Ext.2 and the maximal EOP differences appear for the CPO with a shift of $10.3 \mu$ as in $X$ and drift of $0.9 \mu$ as year ${ }^{-1}$ in $Y$, close to the values reported in Table 3 of Heinkelmann et al. (2015) for the period 1984 to 2013 .
Table 6 EOP Differences ( $\mu$ as or $\mu$ s for dUT1) between EOP estimated with ICRF2 and ICRF1 ext. 2

\begin{tabular}{|c|c|c|c|}
\hline \multirow[t]{2}{*}{ EOP } & \multicolumn{3}{|c|}{ Case 2 vs. case 1} \\
\hline & Shift & Drift & WRMS \\
\hline$\Delta x_{\mathrm{pol}}(\mu \mathrm{as})$ & $5.0 \pm 3.1$ & $0.5 \pm 0.2$ & 51.1 \\
\hline$\Delta y_{\mathrm{pol}}(\mu \mathrm{as})$ & $-7.9 \pm 2.5$ & $0.5 \pm 0.2$ & 41.3 \\
\hline$\Delta \mathrm{dUT} 1(\mu \mathrm{s})$ & $1.0 \pm 0.1$ & $0.0 \pm 0.01$ & 1.6 \\
\hline$\Delta X(\mu$ as $)$ & $-10.3 \pm 2.6$ & $-0.7 \pm 0.1$ & 45.5 \\
\hline$\Delta Y(\mu$ as $)$ & $4.1 \pm 3.2$ & $-0.9 \pm 0.2$ & 56.4 \\
\hline
\end{tabular}

Shift (referred at epoch J2000.0) and linear trends (year ${ }^{-1}$ ) are estimated by WLS. The WRMS are computed after subtracting the linear component of the difference

\subsection{Similarity transformation vs. VLBI ERP differences}

Going back to the tests performed in Sect. 3.3 and recalling the comments relative to the ITRF2005 case, it seems clear that those results show that the differences among EOPs derived using distinct ITRFs cannot be explained simply by the nominal Helmert transformation between the implied frames. This could be surprising at first glance, but our analysis is performed with the series of VLBI individual sessions. Each session involves a small number of stations compared with the number of defining ITRF stations and their geographical distribution is not homogeneous at all. It makes sense to consider separately the sub-networks of stations participating at each session to define a suitable epochframe associated specifically to each session. To investigate whether the EOP differences determined in the previous Sect. 3.3 can be attributed to the differences in orientation of those particular frames to some extent, the corresponding six Helmert transformation parameters were estimated per each VLBI session using WLS. We computed the transformation parameters of the various frames given in Table 2 w.r.t. ITRF2008 for each individual station subset of the included VLBI session: three translation components, and three rotation angles, designated, $T_{x}, T_{y}, T_{z}, R_{1}, R_{2}$, and $R_{3}$, respectively. The scale factor is not determined to be consistent with the VLBI estimates, which have been calculated fixing the station coordinates. Equation (3) shows the similarity transformation applied, where $x_{i}, y_{i}$, and $z_{i}$ are the Cartesian coordinates of the $i$-th point common in the two reference frames, ITRF2008 and each considered alternative TRF:

$$
\left(\begin{array}{c}
x_{i} \\
y_{i} \\
z_{i}
\end{array}\right)_{\mathrm{ITRF} 2008}=\left(\begin{array}{c}
T_{x} \\
T_{y} \\
T_{z}
\end{array}\right)+R\left(\begin{array}{c}
x_{i} \\
y_{i} \\
z_{i}
\end{array}\right)_{\mathrm{TRF}} .
$$

Let us insist that this transformation is between subnetworks, not between the relevant TRFs, although we use an 
abridged notation that makes no reference to sessions. The transformation parameters per each VLBI session were estimated after each subset of station coordinates was brought at the same VLBI epoch using its own station motion model (considering the a priori catalogue positions and velocities) as performed in Feissel et al. (1993). To make sure that each transformation was consistent with the VLBI estimates, each Helmert estimate was computed with the same stations that appear in each VLBI session included in the analyses of Sect. 3.3. For the comparison, the derived ERP (Sect. 3.3) were expressed as global rotations using

$R_{1}=-y_{\mathrm{pol}} \quad R_{2}=x_{\mathrm{pol}} \quad R_{3}=r^{\prime} \cdot \mathrm{dUT} 1-\mathrm{A}_{3}$

where we assumed the rotational contribution from the ICRF to dUT1 to be negligible $\left(A_{3}=0\right)$ due to the relative insensitivity of $\mathrm{d} X$ and $\mathrm{d} Y$ to TRF changes described in Sect. 3.3. Here, $r^{\prime} \approx 0.997$ denotes the ratio between solar and sidereal time.

Table 7 summarizes the statistics of the differences between estimated similarity transformation parameters and estimated VLBI ERP differences for each TRF of Table 5; they are expressed as global rotations in both the cases and referred to ITRF2008 in terms of their relative linear regressions and standard deviations (STD) of their differences. Differences are always computed, so that the ERP series estimated from ITRF2008 are the minuend.

The most relevant results are: (1) ITRF2000 presents considerable drift for $R_{2}\left(-17.4 \mu\right.$ as year $\left.{ }^{-1}\right)$ and shifts in all the rotations; (2) between ITRF2005 and ITRF2008, there are still significant shifts. This is astonishing, because ITRF2008 orientation and orientation stability are defined by no-net rotation (NNR) with respect to ITRF2005. Obviously, the NNR condition (kinematically non-rotation) that is based on a subset of stations common for ITRF2005 and ITRF2008 does not exactly force non-rotation for another subset of stations, such as the VLBI station subset, used in our study; (3) another interesting results are found for the VTRF2008 case, with large $\operatorname{drift}\left(19.9 \mu\right.$ as year $\left.^{-1}\right)$ for $R_{1}$; (4) DTRF2008 shows a large shift (173.0 $\mu$ as) for dUT1; (5) drifts and shifts are very similar in both approaches; and (6) for DTRF2008 and VTRF2008, the STD are about $20 \mu$ as probably caused by an incomplete atmosphere modeling and inaccuracies of the station coordinates; and for ITFR2005 and ITFR2000, the STD are larger, reaching around $50 \mu$ as in the last case. The smallest STD can be found comparing ITRF2008 to DTRF2008. Let us recall that the results labelled as Helmert trans. in Table 7 do not involve VLBI data, whereas the block labelled as VLBI is computed from EOP VLBI solutions. The similarity of shifts and drifts (pointed in 5) and the magnitude of the STD (described in 6) prove that the VLBI data analysis can work as an accurate tool to determine frame inconsistencies.

\section{Summary and conclusions}

An experimental study has been carried out addressing the consistency of the current conventional reference frames (ITRF2008 and ICRF2) and the associated IERS 08 C04 series. Since we are concerned with all five EOP, the only technique that can be applied is VLBI.

Concerning the study on the effects of the unmodeled geophysical signals conducted in Sect. 3.1, one can say that these neglected signals induce damaging effects on the terrestrial pole coordinates and dUT1, causing a strong decrease of the correlation among EOP based on fixed coordinates and EOP based on adjusted coordinates with a priori from ITRF2008. Maximal differences affect $y_{\text {pol }}$ and are a $26.9 \mu$ as shift and a $2.1 \mu$ as year ${ }^{-1}$ drift (Table 3 ). These values give an idea of how good the ITRF2008 is.

The EOP estimated by VLBI analysis might, in addition, depend on the choice of the a priori EOP, and thus, we investigated that possibility. Here, we compare EOP adjustment with respect to IERS 08 C04 when using IERS 08 C04, USNO finals, or the astronomical conventional precession/nutation models, IAU2006/2000A. The comparison between EOP estimated using IERS $08 \mathrm{C} 04$ and USNO finals exhibits a large scatter of dUT1 at the level of $5.3 \mu \mathrm{s}$ (Table 4).

VTRF2008 is consistent with ICRF-Ext. 2 which contains precise positions of more than 3000 compact radio astronomical sources. Different EOP series are estimated with identical VLBI solutions, but with different celestial reference frames (ICRF2 and ICRF-Ext. 2) to analyze their mutual stability. The maximal EOP differences $(10.3 \mu$ as at $\Delta X$ and $0.9 \mu$ as year $^{-1}$ at $\Delta Y$ ) (Table 6) fulfill the stability goal for celestial pole offsets of about $10 \mu$ as. Therefore, the ICRF2 and ICRF-Ext. 2 orientations can be assumed identical within this uncertainty, which is below the threshold of accuracy targeted by GGOS and associated working groups.

However, when the EOP are estimated by fixing station positions on various terrestrial reference frames, serious inconsistencies are detected with respect to ITRF2008 (Table 7). ITRF2000 and ITRF2005 are included in this comparison, because the orientation of ITRF2008 is realized by NNR conditions with respect to the orientation of the ITRF2005, which in its turn is realized via NNR condition with respect to ITRF2000. Although the ITRF2008 and the ITRF2005 are constrained to be kinematically non-rotating with an uncertainty of $8 \mu$ as and $8 \mu$ as year ${ }^{-1}$, meaningful differences above this level and even larger discrepancies with respect to ITRF2000 are found (in particular in the terrestrial pole coordinates) based on the VLBI subset of stations. A marked inconsistency is the differential drift in $y_{\text {pol }}$ of about $-19.9 \mu$ as year ${ }^{-1}$, between VTRF2008 and ITRF2008; it means that ICRF2, ITRF2008, and the conventional EOP series are not completely consistent. ITRF2000 shows large 
Table 7 Global rotations $\left(R_{1}, R_{2}\right.$, and $R_{3}$ ) for each VLBI session from similarity transformation and ERP differences between ITRFs w.r.t. ITRF2008 based VLBI solutions

\begin{tabular}{|c|c|c|c|c|c|}
\hline & \multicolumn{2}{|c|}{ Helmert trans. ( $\mu$ as) } & \multicolumn{2}{|l|}{ VLBI ( $\mu$ as) } & \multirow{2}{*}{$\begin{array}{l}\text { Comparison ( } \mu \text { as) } \\
\text { STD }\end{array}$} \\
\hline & Shift & Drift & Shift & Drift & \\
\hline \multicolumn{6}{|c|}{ ITRF2005 } \\
\hline$R 1$ & $20.9 \pm 9.4$ & $8.8 \pm 0.6$ & $30.9 \pm 8.9$ & $8.9 \pm 0.6$ & 26.0 \\
\hline$R 2$ & $21.3 \pm 6.6$ & $-7.9 \pm 0.4$ & $12.2 \pm 6.3$ & $-7.8 \pm 0.4$ & 29.3 \\
\hline$R 3$ & $121.4 \pm 4.7$ & $-8.9 \pm 0.3$ & $108.4 \pm 4.3$ & $-8.1 \pm 0.3$ & 25.0 \\
\hline \multicolumn{6}{|c|}{ DTRF2008 } \\
\hline$R 1$ & $-1.0 \pm 1.3$ & $0.2 \pm 0.1$ & $8.7 \pm 1.3$ & $-0.4 \pm 0.1$ & 20.7 \\
\hline$R 2$ & $-36.2 \pm 0.8$ & $1.9 \pm 0.1$ & $-31.3 \pm 1.1$ & $1.9 \pm 0.1$ & 18.1 \\
\hline$R 3$ & $172.7 \pm 0.7$ & $-3.1 \pm 0.1$ & $173.0 \pm 0.9$ & $-3.0 \pm 0.1$ & 13.5 \\
\hline \multicolumn{6}{|c|}{ VTRF2008 } \\
\hline$R 1$ & $19.8 \pm 1.7$ & $19.8 \pm 0.1$ & $27.9 \pm 1.5$ & $19.9 \pm 0.1$ & 21.8 \\
\hline$R 2$ & $47.1 \pm 1.5$ & $1.6 \pm 0.1$ & $48.9 \pm 1.4$ & $1.2 \pm 0.1$ & 21.4 \\
\hline$R 3$ & $96.3 \pm 0.9$ & $-0.5 \pm 0.1$ & $92.9 \pm 0.9$ & $-1.3 \pm 0.1$ & 16.7 \\
\hline \multicolumn{6}{|c|}{ ITRF2000 } \\
\hline$R 1$ & $112.9 \pm 9.0$ & $5.7 \pm 0.7$ & $123.5 \pm 8.3$ & $6.1 \pm 0.6$ & 50.3 \\
\hline$R 2$ & $-77.2 \pm 8.2$ & $-17.8 \pm 0.6$ & $-89.6 \pm 8.9$ & $-17.4 \pm 0.7$ & 51.4 \\
\hline$R 3$ & $80.0 \pm 4.2$ & $-1.1 \pm 0.3$ & $80.0 \pm 4.8$ & $-1.9 \pm 0.4$ & 41.0 \\
\hline
\end{tabular}

Shifts (referred to epoch J2000.0) and linear drifts (year ${ }^{-1}$ ) are estimated by LS

The comparison between these two approaches is made by the standard deviation (STD) of the EOP differences shifts in $x_{\mathrm{pol}}$ and $y_{\mathrm{pol}}$, and a considerable drift of about 17.4 $\mu$ as year ${ }^{-1}$ in $x_{\mathrm{pol}}$. Besides, dUT1 evidences substantial inconsistency problems in all tested TRFs, the most pronounced results being between ITRF2008 and DTRF2008 (more than $5 \mathrm{~mm}$ at the Earth equator). These detrimental effects could come from unconsidered geophysical signals (e.g., non-tidal ocean loading), which are neglected and need to be identified. In contrast, celestial pole coordinates, in all the cases, do not show noticeable systematic effects.

Summarizing, in the last 30 years, the EOP accuracy has reached levels, where the margin of improvement is extremely limited. In spite of this enhancement, our study confirms the conclusion that neither the IERS EOP series nor the ITRFs considered in our tests are accurate enough to meet the GGOS goals.

In spite of the valuable advances along many years, the consistency resulting from the combination process continues being at least debatable, considering the extreme differences of the weights assigned to the solutions depending on the various techniques and EOP. Namely, the International GNSS Service (IGS) solution contributes to the ERP by about $95 \%$, but nothing to the offsets of the Celestial Intermediate Pole, which is based only on VLBI results. The latter are ignored in the combination and added later for the sake of completeness. Moreover, the orientation of the VLBI ground network that refers to the ICRF via the VLBI EOP is allowed to rotate during the combination. Consequently, we can infer that the IERS $08 \mathrm{C} 04$ do not refer exactly to
ICRF. Other potential causes of inconsistency are the current methodology of inheriting the orientation from the previous realization to the current realization by applying the NNR condition, i.e., the new frame inherits the "errors" of all its predecessors and adds its own errors. Inconsistencies are also due to the misfit of the true station coordinates and the simple coordinate model used for its approximation, i.e., the uncorrected non-linear station displacement will propagate into EOP, since non-linear effects are not removed. These causes could be among the reasons why the optimum consistency level is not being accomplished yet. Proposals to achieve higher accuracy and consistency are that the ITRF, the EOP, and the ICRF have to be determined in one monolithic adjustment, including all observations of all involved techniques (VLBI, DORIS, GNSS, and SLR), the usage of epoch reference frames (Bloßfeld et al. 2014), and extending the TRF coordinate model to include seasonal signals, as it is foreseen for the next realization of ITRS, ITRF2014 (Altamimi et al. 2016).

Acknowledgments This work has been partly supported by two Spanish Projects from CGL2010-12153-E, AYA2010-22039-C02-01, and AYA2010-22039-C02-02.

\section{References}

Altamimi Z, Sillard P, Boucher C (2002) ITRF2000: A new release of the International Terrestrial Reference Frame for earth science appli- 
cations. J Geophys Res 107:ETG 2-1 ETG 2-19. doi:10.1029/ 2001JB000561

Altamimi Z, Collilieux X, Legrand J, Garayt B, Boucher C (2007) ITRF2005: A new release of the International Terrestrial Reference Frame based on time series of station positions and Earth Orientation Parameters. J Geophys Res 112(B09):401. doi:10.1029/ 2007JB004949

Altamimi Z, Collilieux X, Metivier L (2011) ITRF2008: an improved solution of the International Terrestrial Reference Frame. J Geod 85:457-473. doi:10.1007/s00190-011-0444-4

Altamimi Z, Dermanis A (2012) The choice of reference system in ITRF formulation. In: Sneeuw N et al (eds) VII Hotine-Marussi Symposium on Mathematical Geodesy, International Association of Geodesy, Symposia 137. Springer, Berlin, pp 329-334

Altamimi Z, Rebischung P, Métivier L, Collilieux X (2016) ITRF2014: A new release of the International Terrestrial Reference Frame modeling non-linear station motions. J Geophys Res (under review)

Belda S, Ferrándiz JM, Heinkelmann R, Nilsson T, Schuh H (2016) Testing a New Free Core Nutation empirical model. J Geodyn. doi:10.1016/j.jog.2016.02.002

Bizouard C, Gambis D (2011) The combined solution C04 for Earth Orientation Parameters consistent with International Terrestrial Reference Frame 2008. Technical Note. http://hpiers.obspm.fr/ iers/eop/eopc04/C04.guide.pdf

Bloßfeld M, Seitz M, Angermann D (2014) Non-linear station motions in epoch and multi-year reference frames. J Geod 88:45-63. doi:10.1007/s00190-013-0668-6

Böckmann S, Artz T, Nothnagel A (2010) VLBI terrestrial reference frame contributions to ITRF2008. J Geod 84:201-219. doi:10. 1007/s00190-009-0357-7

Böhm J, Werl B, Schuh H (2006) Troposphere mapping functions for GPS and very long baseline interferometry from European Centre for Medium-Range Weather Forecasts operational analysis data. J Geophy Res 111:B2. doi:10.1029/2005JB003629

Böhm J, Böhm S, Pany A, Plank L, Spicakova H, Teke T, Schuh H (2012) The New Vienna VLBI Software VieVS. Geod for Planet Earth. Proc 2009 IAG Symp:1007-1011. doi:10.1007/ 978-3-642-20338-1126

Chao B, Hsieh Y (2015) The earths free core nutation: Formulation of dynamics and estimation of eigenperiod from the very-longbaseline interferometry data. Earth Planet Sci Lett 36:483-492

Dehant V (2002) Report of the IAU working group on 'Non-rigid Earth Nutation Theory'. Highlights Astron 12:117-119

Dick R (2013) IERS Annual Report 2011. International Earth Rotation and Reference Systems Service, Central Bureau. Frankfurt am Main: Verlag des Bundesamts für Kartographie und Geodäsie, p 159. ISBN:978-3-86482-046-5

Ding XL, Zheng DW, Dong DN, Ma C, Chen YQ, Wang GL (2005) Seasonal and secular positional variations at eight collocated GPS and VLBI stations. J Geod 79:71-81. doi:10.1007/ s00190-005-0444-3

Eriksson D, MacMillan DS (2014) Continental hydrology loading observed by VLBI measurements. J Geod 88:675-690. doi:10. 1007/s00190-014-0713-0

Ferrándiz JM, Gross R (2014) The New IAU/IAG Joint Working Group on Theory of Earth Rotation. Proc IAG Symp 143 (to appear)

Ferrándiz JM, Belda S, Heinkelmann R, Getino J, Schuh H (2016) Reference frames in earth rotation theories (to be submitted)

Feissel M, Bourquard D, Charlot P, Eisop E, Essaifi N, Lestrade JF, Arias EF, Boucher C, Altamimi Z (1993) Earth Orientation and Related Reference Frames. In: Smith DE, Turcotte DL (eds) Contributions of space geodesy to geodynamics: earth dynamics. American Geophysical Union, Washington, D.C. doi:10.1029/GD024p0099

Fey AL, Ma C, Arias EF, Charlot P, Feissel-Vernier M, Gontier AM, Jacobs CS, Li J, Macmillan DS (2004) The Second Extension of the International Celestial Reference Frame: ICRF-EXT.1. Astron J 127:3587-3608. doi:10.1086/420998

Fey AL, Gordon D, Jacobs CS, Ma C, Gaume RA, Arias EF, Bianco G, Boboltz DA, Boeckmann S, Bolotin S, Charlot P, Collioud A, Engelhardt G, Gipson J, Gontier AM, Heinkelmann R, Kurdubov S, Lambert S, Lytvyn S, MacMillan DS, Malkin Z, Nothnagel A, Ojha R, Skurikhina E, Sokolova J, Souchay J, Sovers OJ, Tesmer V, Titov O, Wang G, Zharov V (2015) The Second Realization of the International Celestial Reference Frame by very long baseline interferometry. Astron J 150:58. doi:10.1088/0004-6256/150/2/ 58

Heinkelmann R, Nilsson T, Karbon M, Liu L, Lu C, MoraDiaz JA, Parselia E, Raposo-Pulido V, Soja B, Xu M, Schuh H (2014a) The GFZ VLBI Solution: Characteristics and First Results. In: Behrend D, Baver KD, Armstrong $\mathrm{K}$ (eds) Proc of the Eighth IVS General Meeting: VGOS: The New VLBI Network ftp://ivscc.gsfc.nasa.gov/pub/generalmeeting/2014/IVS-2014-General-Meeting-Proceedings.pdf

Heinkelmann R, Belda S, Ferrándiz JM, Schuh H (2014b) The Consistency of The Current Conventional Celestial and Terrestrial Reference Frames and the Conventional EOP Series. In: Malkin Z, Capitaine N (eds) Proc of the Journées 2014 "Systèmes de référence spatio-temporels": Recent developments and prospects in ground-based and space astrometry, St. Petersburg, Russia, pp 224-225. ISBN:978-5-9651-0873-2, ISBN:978-2-901057-70-3

Heinkelmann R, Belda S, Ferrándiz JM, Schuh H (2015) How Consistent are The Current Conventional Celestial and Terrestrial Reference Frames and The Conventional Earth Orientation Parameters? In: Rizos C, Willis P (eds) Proceedings of the IAG Commision 1 Symposium 2014: Reference Frames for Applications in Geosciencies (International Association of Geodesy Symposia). Springer, Berlin. doi:10.1007/1345_2015_149

Hilton J, Capitaine N, Chapront J, Ferrándiz JM, Fienga A, Fukushima T, Getino J, Mathews P, Simon JL, Soffel M, Vondrak J, Wallace P, Williams J (2006) Report of the International Astronomical Union Division I Working Group on Precession and the Ecliptic. Celest Mech Dyn Astron 94:351-367. doi:10.1007/s10569-006-0001-2

Krásná H, Böhm J, Schuh J (2013) Free Core Nutation observed by VLBI. Astron Astrophys 555:A29. doi:10.1051/0004-6361/ 201321585

Krásná H, Böhm J, Plank L, Nilsson T, Schuh H (2014) Atmospheric effects on VLBI-derived terrestrial and celestial reference frames. In: Rizos C, Willis P (eds) Earth in the edge: science for a sustainable planet. IAG Symp, vol 139. Springer, Berlin, pp 203-207. doi: $10.1007 / 978-3-642-37222-3 \_26$

Krásná H, Malkin Z, Böhm J (2015) Non-Linear VLBI station motions and their impact on the celestial reference frame and Earth orientation parameters. J Geod 89. doi:10.1007/s00190-015-0830-4

Lambert S (2007) Empirical Model of the Earth's Free Core Nutation. Technical note. http://syrte.obspm.fr lambert/fcn/

Ma C, Arias EF, Eubanks T, Fey A, Gontier AM, Jacobs C, Archinal OSB, Charlot P (1998) The International Celestial Reference Frame as Realized by Very Long Baseline Interferometry. Astron J 116:516. doi: $10.1086 / 300408$

$\mathrm{Ma} C$ et al (2009) The Second Realization of the International Celestial Reference Frame by Very Long Baseline Interferometry. In: Fey AL, Gordon D, Jacobs CS (eds) IERS Technical Note, No. 35. Verlag des Bundesamts für Kartographie und Geodäsie, Frankfurt am Main, pp 1-204

Malkin Z (2010) Comparison of CPO and FCN empirical models. In: Capitaine N (ed) Proc Journ 2010: New challenges for reference systems and numerical standards in astronomy, Paris, France, pp $172-175$

Malkin Z (2013a) Impact of seasonal station motion on VLBI UT1 intensives results. J Geod 87:505-514. doi:10.1007/ s00190-013-0624-5 
Malkin Z (2013) Free core nutation and geomagnetic jerks. J. Geodyn 72:53-58. doi:10.1016/j.jog.2013.06.001

Nilsson T, Heinkelmann R, Karbon M, Raposo-Pulido V, Soja B, Schuh H (2014) Earth orientation parameters estimated from VLBI during the CONT11 campaign. J Geod 88:491-502. doi:10.1007/ s00190-014-0700-5

Petit G, Luzum B (2010) IERS Technical Note 36, vol 179. Verlag des Bundesamts für Kartographie und Geodäsie, Frankfurt am Main, p 179. ISBN:3-89888-989-6

Plag H, Pearlman M (2009) Global Geodetic Observing System: Meeting the Requirements of a Global Society on a Changing Planet in 2020. Springer, New York, p 332. doi:10.1007/ 978-3-642-02687-4
Seitz M, Angermann D, Blo $\beta$ feld M, Drewes H, Gerstl M (2012) The 2008 DGFI realization of the ITRS: DTRF2008. J Geod 86:10971123. doi:10.1007/s00190-012-0567-2

Tesmer V, Steigenberger P, Rothacher M, Boehm J, Meisel B (2009) Annual deformation signals from homogeneously reprocessed VLBI and GPS height time series. J Geod 83:973-988. doi:10. 1007/s00190-009-0316-3 


\section{Author Query Form}

\section{Please ensure you fill out your response to the queries raised below and return this form along with your corrections}

\section{Dear Author}

During the process of typesetting your article, the following queries have arisen. Please check your typeset proof carefully against the queries listed below and mark the necessary changes either directly on the proof/online grid or in the 'Author's response' area provided below

\begin{tabular}{|c|c|c|}
\hline Query & Details required & Author's response \\
\hline 1. & $\begin{array}{l}\text { Kindly check and confirm that Table } 1 \mathrm{a} \text { and } \mathrm{b} \text { is splitted } \\
\text { into Tables } 1 \text { and } 2 \text { and also check and confirm the } \\
\text { change made in Table } 4 .\end{array}$ & \\
\hline 2. & $\begin{array}{l}\text { Kindly check and update these references "Altamimi } \\
\text { et al. (2016); Heinkelmann et al. (2014a, b)". }\end{array}$ & \\
\hline
\end{tabular}

